\title{
A MASS TRANSPORTATION APPROACH FOR SOBOLEV INEQUALITIES IN VARIABLE EXPONENT SPACES
}

\author{
JUAN PABLO BORTHAGARAY, JULIÁN FERNÁNDEZ BONDER AND ANALÍA SILVA
}

\begin{abstract}
In this paper we provide a proof of the Sobolev-Poincaré inequality for variable exponent spaces by means of mass transportation methods, in the spirit of [4]. The importance of this approach is that the method is flexible enough to deal with different inequalities. As an application, we also deduce the Sobolev-trace inequality improving the result of [11] by obtaining an explicit dependence of the exponent in the constant.
\end{abstract}

\section{INTRODUCTION}

The goal of this paper is to show how mass transportation techniques can be applied to prove Sobolev inequalities in the context of variable exponent spaces.

Mass transportation is a subject that originates in the work of Monge in the XVIII century (cf. [15]) and was mathematically stated in modern terms in the work of Kantorovich in the 1940s, [13].

This topic has experienced a revolution since the by now classical paper of Brenier in 1987 (see $[2,3])$. It is by now almost impossible to give a complete list of references or even topics where mass transportation methods are applied. We refer to the excellent books of Villani [17, 18].

The application of mass transportation methods to Sobolev inequalities was first made by Cordero-Erausquin, Nazaret and Villani in [4]. See also [16] where the trace inequality was studied.

The first inequality that we are going to deal with is the Sobolev-Poincaré inequality.

Given a measurable function $p: \mathbb{R}^{n} \rightarrow \mathbb{R}$ such that

$$
1 \leq p_{-}:=\inf p \leq p_{+}:=\sup p<n
$$

(here and throughout the paper, by inf and sup we mean the essential infimum and the essential supremum respectively), the Sobolev-Poincaré inequality states the existence of a constant $C>0$ such that

$$
\|f\|_{p^{*}} \leq C\|\nabla f\|_{p}
$$

for every $f \in C_{c}^{\infty}\left(\mathbb{R}^{n}\right)$, where $p^{*}=\frac{n p}{n-p}$ and the norms are the so-called Luxemburg norms associated to the modular

$$
\rho_{r}(f):=\int_{\mathbb{R}^{n}}|f|^{r} d x
$$

defined for any variable exponent $r: \mathbb{R}^{n} \rightarrow[1, \infty)$.

2010 Mathematics Subject Classification. 46E35,49J40.

Key words and phrases. Sobolev inequalities, variable exponents, mass transportation. 
That is,

$$
\|f\|_{p^{*}}:=\inf \left\{\lambda>0: \rho_{p^{*}}\left(\frac{f}{\lambda}\right) \leq 1\right\}, \quad\|\nabla f\|_{p}:=\inf \left\{\lambda>0: \rho_{p}\left(\frac{|\nabla f|}{\lambda}\right) \leq 1\right\}
$$

The validity of (1.2) for constant exponents $p$ is well-known and we refer to the book of Adams, [1].

For variable exponents, the validity of $(1.2)$ was established in $[5,7,8,12]$. See $[6$, Theorem 8.3.1] for a proof.

The hypotheses on $p$ for (1.2) to hold are, in addition to (1.1), that $p$ be globally log-Hölder continuous. See [6, Chapter 4] for the definition of log-Hölder continuity.

Moreover, in [6, Theorem 8.3.1], it is shown that the constant $C$ in (1.2) depends only con $n, p_{+}$and the log-Hölder constant of $p$ denoted by $c_{\log }(p)$.

The proof in [6] is based on Harmonic Analysis techniques. More precisely, they use the boundedness of the Hardy-Littlewood maximal function and of the Riesz potentials in variable exponent spaces.

The mass transportation approach to this problem, is more direct and elementary. The only technical result that is needed is Brenier's theorem that asserts the existence of a transport $T$ between two probability measures and that this transport is the gradient of a convex function. This approach is the same that was used by Cordero-Erausquin, Nazaret and Villani in [4] dealing with the constant exponent case. See next section for the details.

Even though this method provides a more elementary proof of the Sobolev-Poincaré inequality (1.2), the main drawback is that we end up with a more restrictive hypotheses on the exponent $p$. We require $p$ to be weakly differentiable. More precisely, we need to ask that $p \in W^{1, s}\left(\mathbb{R}^{n}\right)$, for some $s>n$. Moreover, the constant in (1.2) that appears in our proof also depends on the support of $f$. More precisely, it depends on the diameter of the support.

Our result for the Sobolev-Poincaré inequality reads

Theorem 1.1. Assume that $\nabla p \in L^{s}\left(\mathbb{R}^{n}\right)$ for some $s>n$.

Then, there exists constants $C\left(c, p_{+}, R\right), C\left(n, p_{+}\right), \gamma\left(n, p_{+}\right)>0$ such that the following SobolevPoincaré inequality holds

$$
\|f\|_{p^{*}} \leq C\left(n, p_{+}, R\right)\|\nabla p\|_{s}^{\gamma\left(n, p_{+}\right)} \max \left\{\|\nabla f\|_{p}^{\frac{1}{\left(\frac{s}{s-p}\right)_{+}}},\|\nabla f\|_{p}^{\frac{1}{\left(\frac{s}{s-p}\right)_{-}}}\right\}^{\frac{1}{p_{+}}}+C\left(n, p_{+}\right)\|\nabla f\|_{p}
$$

for any $f \in C_{c}^{\infty}\left(\mathbb{R}^{n}\right)$ such that $\operatorname{supp} f \subset B_{R}$.

Nevertheless, since the proof is simpler, it is flexible enough to deal with other inequalities. As an example, in Section 3, we treat the Sobolev trace inequality,

$$
\|f\|_{p_{*}, \mathbb{R}^{n-1}} \leq C\|\nabla f\|_{p, \mathbb{R}_{+}^{n}}
$$

for every $f \in C_{c}^{\infty}\left(\mathbb{R}^{n}\right)$ with $C>0$ depending on $n, p_{+},\|\nabla p\|_{s}$ and the support of $f$, where $p_{*}=\frac{(n-1) p}{n-p}$ and the (Luxemburg) norms are defined analogously as before.

The proof of this inequality in the constant exponent case is classical (see again the book of Adams [1]). Also recall that mass transportation methods were applied in the constant exponent case for the Sobolev trace inequality (1.4) by Nazaret in [16]. Our proof follows closely the one in $[16]$. 
Our result reads

Theorem 1.2. Let $p \in W^{1, s}\left(\mathbb{R}_{+}^{n}\right)$ for some $s>n$, be an exponent satisfying (1.1). Then, there exists some constant $C=C\left(n, p_{+}, p_{-}, s,\|\nabla p\|_{s, \mathbb{R}_{+}^{n}}, R\right)$ such that

$$
\|f\|_{p_{*}, \mathbb{R}^{n-1}} \leq C\|\nabla f\|_{p, \mathbb{R}_{+}^{n}}
$$

for every $f \in C_{c}^{\infty}\left(\mathbb{R}^{n}\right)$ such that $\operatorname{supp} f \subset B_{R}$.

The variable exponent case of (1.4) was proved by Fan in [11]. Here we recover Fan's result and, moreover, by our method we can give the precise dependence of $C$ on the regularity of the exponent $p$ that was missing in [11].

Organization of the paper. After this short introduction, the paper is divided into two sections. Section 2 deals with the Sobolev-Poincaré inequality (1.2) and Section 3 deals with the Sobolev trace inequality (1.4).

\section{The Sobolev-Poincaré Inequality}

As we mentioned in the introduction, the mass transportation approach to the proof of the Sobolev-Poincaré inequality (1.2) follows the lines of the paper by Cordero-Erausquin, Nazaret, Villani [4]. We will point out the differences in the arguments when they arrive.

First, observe that is enough to prove (1.2) for nonnegative functions $f \in C_{c}^{\infty}\left(\mathbb{R}^{n}\right)$. So, let $f, g \in C_{c}^{\infty}\left(\mathbb{R}^{n}\right)$ be nonnegative functions such that $\|f\|_{p^{*}}=\|g\|_{p^{*}}=1$ and define the probability densities $F=f^{p^{*}}$ and $G=g^{p^{*}}$.

According to Brenier's theorem, $[2,3]$ (see $[17$, Theorem 3.8] for a proof), there exists a convex lower semi-continuous function $\varphi$ such that $\nabla \varphi$ transports the measure $d \mu=F d x$ (optimally) to $d \nu=G d x$.

This implies, in particular, that for all $\psi \in L^{1}(d \nu)$ the following transport identity is valid:

$$
\int_{\mathbb{R}^{n}}(\psi \circ \nabla \varphi) d \mu=\int_{\mathbb{R}^{n}} \psi d \nu
$$

Moreover, the following Monge-Ampère equation

$$
F(x)=G(\nabla \varphi(x)) \operatorname{det}\left(D_{A}^{2} \varphi(x)\right)
$$

holds $\mu$-a.e., where $D_{A}^{2} \varphi$ is the Alexandrov Hessian of $\varphi$. See [14].

Moreover, $\nabla \varphi$ is invertible. In fact

$$
(\nabla \varphi)^{-1}=\nabla \varphi^{*}
$$

where $\varphi^{*}$ is the Legendre transform of $\varphi$. See [17].

Let us recall that the Alexandrov Hessian of a convex function $\varphi$ is the absolute continuous part of the distributional Hessian of $\varphi$ and that by Alexandrov's Theorem, it coincides with the Hessian of $\varphi$ a.e. with respect to Lebesgue's measure. See [10, Chapter 6].

Let us define the exponent

$$
p_{*}=\frac{(n-1) p}{n-p}=\left(1-\frac{1}{n}\right) p^{*} .
$$


Therefore, by (2.1) and (2.2),

$$
\int_{\mathbb{R}^{n}} g^{p_{*}} d x=\int_{\mathbb{R}^{n}} G^{1-\frac{1}{n}} d x=\int_{\mathbb{R}^{n}}\left(G^{-\frac{1}{n}} \circ \nabla \varphi\right) d \mu=\int_{\mathbb{R}^{n}} F^{1-\frac{1}{n}} \operatorname{det}\left(D_{A}^{2} \varphi\right)^{\frac{1}{n}} d x .
$$

Since the distributional Hessian of $\varphi$ is nonnegative (recall that $\varphi$ is convex), it holds that $D_{A}^{2} \varphi \leq D^{2} \varphi$ in the distributional sense. Thus, by applying first the arithmetic-geometric inequality for nonnegative symmetric matrices and integrating by parts we obtain

$$
\int_{\mathbb{R}^{n}} g^{p_{*}} d x \leq \frac{1}{n} \int_{\mathbb{R}^{n}} F^{1-\frac{1}{n}} \Delta_{A} \varphi d x \leq \frac{1}{n} \int_{\mathbb{R}^{n}} F^{1-\frac{1}{n}} \Delta \varphi d x=-\frac{1}{n} \int_{\mathbb{R}^{n}} \nabla\left(F^{1-\frac{1}{n}}\right) \cdot \nabla \varphi d x .
$$

So far, the argument has been exactly as in Cordero-Erausquin, Nazaret, Villani's paper [4] where the reader can check the missing details in the arguments.

Now is where the difference appear since $p$ is not constant. From now on, assume that $p$ is smooth and that the bounds (1.1) hold. When $p \in W^{1, s}\left(\mathbb{R}^{n}\right)$ the difference in the argument is exactly as in [11, Lemma 2.1]. In order to make the arguments more transparent, we make the computations assuming that $p$ is smooth and leave to the reader the details when $p \in W^{1, s}\left(\mathbb{R}^{n}\right)$.

A straightforward computation gives

$$
\nabla\left(F^{1-\frac{1}{n}}\right)=\frac{(n-1) n f^{p_{*}} \log f}{(n-p)^{2}} \nabla p+p_{*} f^{p_{*}-1} \nabla f .
$$

So we obtain the estimate

$$
\int_{\mathbb{R}^{n}} g^{p_{*}} d x \leq \frac{(n-1)}{n-p_{+}}\left(\frac{1}{n-p_{+}} \int_{\mathbb{R}^{n}} f^{p_{*}}|\log f||\nabla \varphi||\nabla p| d x+\frac{p_{+}}{n} \int_{\mathbb{R}^{n}} f^{p_{*}-1}|\nabla f||\nabla \varphi| d x\right) .
$$

We need to estimate the two integrals on the right-hand side of the previous inequality. Applying Hölder's inequality for variable exponent spaces for both integrals (see [6] for the proof of Hölder's inequality in variable exponent spaces), we obtain

$$
\int_{\mathbb{R}^{n}} f^{p_{*}}|\log f\|\nabla \varphi\| \nabla p| d x \leq 2\|f \log f|\nabla p|\|_{p}\left\|f^{p_{*}-1}|\nabla \varphi|\right\|_{p^{\prime}}
$$

and

$$
\int_{\mathbb{R}^{n}} f^{p_{*}-1}|\nabla f||\nabla \varphi| d x \leq 2\|\nabla f\|_{p}\left\|f^{p_{*}-1}|\nabla \varphi|\right\|_{p^{\prime}}
$$

Recall that $p^{\prime}$ is defined, as usual, as $\frac{1}{p}+\frac{1}{p^{\prime}}=1$.

Remark 2.1. In this point of the argument, we require that $p_{-}>1$. Nevertheless, since the constants entering in the estimates do not depend on $p_{-}$as we will see, it can be easily deduced that the result still holds for $p_{-}=1$.

The modular giving the norm $\left\|f^{p_{*}-1}|\nabla \varphi|\right\|_{p^{\prime}}$ can be expressed as

$$
\int_{\mathbb{R}^{n}}\left(f^{p_{*}-1}|\nabla \varphi|\right)^{p^{\prime}} d x=\int_{\mathbb{R}^{n}}|\nabla \varphi|^{p^{\prime}} F d x=\int_{\mathbb{R}^{n}}|y|^{\left(p^{\prime} \circ \nabla \varphi^{*}\right)} G d y,
$$

where we have used the transport identity (2.1) and (2.3). This term is completely analogous to the one appearing in [4] except that the exponent is not constant and therefore depends on $\varphi$. So we need to bound it independently of $\varphi$.

Let us consider the function $\eta(y)=\max \left\{|y|^{\left(p^{\prime}\right)_{+}},|y|^{\left(p^{\prime}\right)_{-}}\right\}$, where

$$
\left(p^{\prime}\right)_{+}=\sup \frac{p}{p-1}=\left(p_{-}\right)^{\prime} .
$$


Hence, since $\left(p_{*}-1\right) p^{\prime}=p^{*},(2.4)$ translates into

$$
\int_{\mathbb{R}^{n}}\left(f^{p_{*}-1}|\nabla \varphi|\right)^{p^{\prime}} d x \leq \int_{\mathbb{R}^{n}} \eta G d y .
$$

So if we define

$$
\alpha(n, p)=\frac{n-p_{+}}{2(n-1)} \sup \frac{\int_{\mathbb{R}^{n}} g^{p_{*}} d x}{\max \left\{\left(\int_{\mathbb{R}^{n}} \eta g^{p^{*}} d y\right)^{\frac{1}{\left(p^{\prime}\right)}-},\left(\int_{\mathbb{R}^{n}} \eta g^{p^{*}} d y\right)^{\frac{1}{\left(p^{\prime}\right)}+}\right\}},
$$

where the supremum is taken over all nonnegative functions $g \in C_{c}^{\infty}\left(\mathbb{R}^{n}\right)$ such that $\|g\|_{p^{*}}=1$ and recall the relation between the modular and the Luxemburg norm, we prove the following result

Theorem 2.2. Let $f \in C_{c}^{\infty}\left(\mathbb{R}^{n}\right)$ be nonnegative and let $p \in W^{1, s}\left(\mathbb{R}^{n}\right), s>n$, be an exponent that verifies (1.1). Assume that $\|f\|_{p^{*}}=1$.

Then, the following inequality holds:

$$
\alpha(n, p) \leq \frac{1}{n-p_{+}}\|f \log f|\nabla p|\|_{p}+\frac{p_{+}}{n}\|\nabla f\|_{p} .
$$

This last estimate is exactly the one obtained in [4] with the exception of the logarithmic term. Observe that if the logarithmic term is removed from (2.6) then the Sobolev-Poincaré inequality (1.2) is proved by homogeneity.

Remark 2.3. It is immediate to see that the term $\alpha(n, p)$ can be bounded below by a term depending only on $n, p_{+}$and $p_{-}$. Moreover, since the constant does not degenerate when $p_{-}=1$ it can be taken depending only con $n$ and $p_{+}$. i.e. there exists a constant $D\left(n, p_{+}\right)>0$ such that

$$
D\left(n, p_{+}\right) \leq \alpha(n, p)
$$

So, the remaining of the proof will be to bound the logarithmic term by a constant times some norm of $|\nabla p|$.

We begin with a lemma.

Lemma 2.4. Let $f \in C_{c}^{\infty}\left(\mathbb{R}^{n}\right)$ be nonnegative, and $p \in W^{1, s}\left(\mathbb{R}^{n}\right), s>n$, be an exponent such that (1.1) holds. Assume $\|f\|_{p^{*}}=1$. Then,

$$
\int_{\mathbb{R}^{n}} f^{p}|\log f|^{p}|\nabla p|^{p} d x \leq\left(C_{1}(f)+C_{2}\right) \max \left\{\|\nabla p\|_{s}^{p_{-}},\|\nabla p\|_{s}^{p_{+}}\right\},
$$

where

$$
\begin{aligned}
& C_{1}(f)=2 \max \left\{\left(C(n, s) \operatorname{diam}(\operatorname{supp} f)\left\|1_{\text {supp } f}\right\|_{p^{\prime}}\|\nabla f\|_{p}\right)^{\frac{1}{\left(\frac{s}{s-p}\right)_{-}}} ;\right. \\
& \left.\left(C(n, s) \operatorname{diam}(\operatorname{supp} f)\left\|1_{\operatorname{supp} f}\right\|_{p^{\prime}}\|\nabla f\|_{p}\right)^{\frac{1}{\left(\frac{s}{s-p}\right)_{+}}}\right\}, \\
& C_{2}=\frac{2 s(n-1)}{e(s-n)} .
\end{aligned}
$$


Proof. The proof is rather elementary. Let us split the integral into two parts, one where $f \leq 1$ and the other one where $f>1$. First, by Hölder's inequality for variable exponents (see [6]), we have

$$
\int_{\{f \leq 1\}} f^{p}|\log f|^{p}|\nabla p|^{p} d x \leq 2\left\|f^{p}|\log f|^{p} 1_{\{f \leq 1\}}\right\|_{\frac{s}{s-p}}\left\||\nabla p|^{p}\right\|_{\frac{s}{p}} .
$$

Now we need to bound the first norm. Let $r:=\frac{s p}{s-p}$ and observe that $r_{-}>1$. Observe that if $f \leq 1$ one has the control $f|\log f| \leq e^{-1}<1$ and so

$$
f^{r}|\log f|^{r} \leq f^{r_{-}}|\log f|^{r_{-}}=f\left(f^{r_{-}-1}|\log f|^{r_{-}}\right) \leq C\left(r_{-}\right) f
$$

where

$$
C\left(r_{-}\right)=\left(\frac{r_{-}}{e\left(r_{-}-1\right)}\right)^{r_{-}} \leq C(n, s) .
$$

Hence, by Poincaré inequality in $L^{1}$ and Hölder inequality for variable exponents,

$$
\begin{aligned}
\int_{\{f \leq 1\}} f^{r}|\log f|^{r} d x & \leq C(n, s) \int_{\mathbb{R}^{n}} f \\
& \leq C(n, s) 2 \operatorname{diam}(\operatorname{supp} f) \int_{\mathbb{R}^{n}}|\nabla f| d x \\
& \leq C(n, s) 4 \operatorname{diam}(\operatorname{supp} f)\left\|1_{\text {supp } f}\right\|_{p^{\prime}}\|\nabla f\|_{p}
\end{aligned}
$$

On the other hand, it holds

$$
\left\||\nabla p|^{p}\right\|_{\frac{s}{p}} \leq \max \left\{\|\nabla p\|_{s}^{p_{-}},\|\nabla p\|_{s}^{p_{+}}\right\}
$$

and therefore

$$
\int_{\{f \leq 1\}} f^{p}|\log f|^{p}|\nabla p|^{p} d x \leq C_{1}(f) \max \left\{\|\nabla p\|_{s}^{p_{-}},\|\nabla p\|_{s}^{p_{+}}\right\},
$$

where $C_{1}(f)$ is defined according to $(2.9)$.

Now, for $f \geq 1$ we write

$$
f^{p}|\log f|^{p}=f^{r}\left(f^{1-\frac{r}{p}}|\log f|\right)^{p} .
$$

Then, if $r>p$ it is immediate to check that,

$$
f^{1-\frac{r}{p}}|\log f| \leq f^{1-\left(\frac{r}{p}\right)_{-}}|\log f| \leq \frac{1}{e\left(\left(\frac{r}{p}\right)_{-}-1\right)}=: K(r, p) .
$$

In consequence, we obtain

$$
\begin{aligned}
\int_{\{f>1\}} f^{p}|\log f|^{p}|\nabla p|^{p} d x & \leq K(r, p) \int_{\mathbb{R}^{n}} f^{r}|\nabla p|^{p} d x \\
& \leq 2 K(r, p)\left\|f^{r}\right\|_{\frac{s}{s-p}}\left\||\nabla p|^{p}\right\|_{\frac{s}{p}}
\end{aligned}
$$

and if we take $r=p^{*}\left(1-\frac{p}{s}\right)$, which verifies $r>p$ since $s>n$, it follows that $\frac{r s}{s-p}=p^{*}$ and so

$$
\left\|f^{r}\right\|_{\frac{s}{s-p}}=1
$$

Observe that by our choice of $r$ we actually have that

$$
K(r, p) \leq C_{2} .
$$


Therefore, from (2.11) we obtain

$$
\int_{\{f>1\}} f^{p}|\log f|^{p}|\nabla p|^{p} d x \leq C_{2} \max \left\{\|\nabla p\|_{s}^{p_{-}},\|\nabla p\|_{s}^{p_{+}}\right\} .
$$

Putting together (2.10) and (2.12) we conclude the desired result.

Remark 2.5. An immediate consequence of the previous lemma is that the Sobolev inequality holds if $p$ satisfies the condition

$$
\max \left\{\|\nabla p\|_{s^{-}}^{p_{-}},\|\nabla p\|_{s^{+}}^{p_{+}}\right\}<\delta
$$

for some $\delta>0$ small enough.

In fact, (2.6) and (2.8) will give

$$
\alpha(n, p)-\frac{1}{n-p_{+}}\left(C_{2} \delta\right)^{\frac{1}{p+}} \leq C_{1}(f)^{\frac{1}{p+}} \max \left\{\|\nabla p\|_{s}^{p_{-}},\|\nabla p\|_{s}^{p_{+}}\right\}^{\frac{1}{p+}}+\frac{p_{+}}{n}\|\nabla f\|_{p} .
$$

Observe that there exists a constant $B\left(n, p_{+}, R, s\right)>0$ such that, if $\operatorname{supp} f \subset B_{R}$,

$$
C_{1}(f)^{\frac{1}{p_{+}}} \leq B\left(n, p_{+}, R, s\right)\|\nabla f\|_{p}
$$

So, by Remark 2.3, we can choose $\delta=\delta\left(n, p_{+}\right)$such that, if supp $f \subset B_{R}$,

$$
0<\frac{D\left(n, p_{+}\right)}{2} \leq\left(B\left(n, p_{+}, R, s\right) \max \left\{\|\nabla p\|_{s}^{\frac{p_{-}}{p_{+}}},\|\nabla p\|_{s}\right\}+C\left(n, p_{+}\right)\right)\|\nabla f\|_{p},
$$

where $D\left(n, p_{+}\right)$is given by $(2.7)$, and the general inequality for all $f \in C_{c}^{\infty}\left(\mathbb{R}^{n}\right)$ follows by homogeneity.

Condition (2.13) is by no means restrictive; indeed, it is possible to obtain the inequality for any exponent by means of a scaling argument. Given any measurable function $f$ on $\mathbb{R}^{n}$ and $k \geq 1$ consider the function $f_{k}(x)=f\left(\frac{x}{k}\right)$. Then, the following lemma holds

Lemma 2.6. Given $f \in C_{c}^{\infty}\left(\mathbb{R}^{n}\right)$ nonnegative, $p$ a smooth exponent satisfying (1.1) and $r \geq 1$ constant, we have

$$
\begin{gathered}
k^{\frac{n}{p_{+}^{*}}}\|f\|_{p^{*}} \leq\left\|f_{k}\right\|_{p_{k}^{*}} \leq k^{\frac{n}{p_{-}^{*}}}\|f\|_{p^{*}} \\
k^{\frac{n}{p_{+}}-1}\|\nabla f\|_{p} \leq\left\|\nabla f_{k}\right\|_{p_{k}} \leq k^{\frac{n}{p_{-}}-1}\|\nabla f\|_{p} \\
k^{-p_{+}+\frac{n}{r}}\left\||\nabla p|^{p}\right\|_{r} \leq\left\|\left.\nabla p_{k}\right|^{p_{k}}\right\|_{r} \leq k^{-p_{-}+\frac{n}{r}}\left\||\nabla p|^{p}\right\|_{r}
\end{gathered}
$$

Remark 2.7. Since $p \mapsto p^{*}$ is monotone with respect to $p$, it follows that $p_{-}^{*}=\left(p_{-}\right)^{*}$ and therefore, by (1.1), that $p_{-}^{*} \geq 1^{*}=\frac{n}{n-1}$. So in Lemma 2.6 the following upper bounds can be obtained

$$
\begin{aligned}
& \left\|f_{k}\right\|_{p_{k}^{*}} \leq k^{n-1}\|f\|_{p^{*}} \\
& \|\nabla f\|_{p_{k}} \leq k^{n-1}\|\nabla f\|_{p} \\
& \left\|\left|\nabla p_{k}\right|^{p_{k}}\right\|_{s} \leq k^{-1+\frac{n}{s}}\left\||\nabla p|^{p}\right\|_{s}
\end{aligned}
$$


Proof. We will prove inequalities (2.16). Inequalities (2.15) follow in the same way. Take $\mu>0$, then

$$
\begin{aligned}
\int_{\mathbb{R}^{n}}\left(\frac{\left|\nabla f_{k}\right|}{\mu}\right)^{p_{k}} d x & =\int_{\mathbb{R}^{n}}\left(\frac{\left|\frac{1}{k} \nabla f\left(\frac{x}{k}\right)\right|}{\mu}\right)^{p\left(\frac{x}{k}\right)} d x \\
& =\int_{\mathbb{R}^{n}}\left(\frac{\left|\frac{1}{k} \nabla f\right|}{\mu}\right)^{p} k^{n} d y \\
& =\int_{\mathbb{R}^{n}}\left(\frac{|\nabla f|}{\mu k^{1-\frac{n}{p}}}\right)^{p} d y
\end{aligned}
$$

Recall that $k \geq 1$, so that

$$
\int_{\mathbb{R}^{n}}\left(\frac{|\nabla f|}{\mu k^{1-\frac{n}{p_{+}}}}\right)^{p} d y \leq \int_{\mathbb{R}^{n}}\left(\frac{\left|\nabla f_{k}\right|}{\mu}\right)^{p_{k}} d x \leq \int_{\mathbb{R}^{n}}\left(\frac{|\nabla f|}{\mu k^{1-\frac{n}{p_{-}}}}\right)^{p} d y .
$$

Then, choosing $\mu=\|\nabla f\|_{p} k^{-1+\frac{n}{p_{+}}}$, we obtain that

$$
\left\|\nabla f_{k}\right\|_{p_{k}} \geq\|\nabla f\|_{p} k^{-1+\frac{n}{p_{+}}} .
$$

Analogously, it holds that

$$
\left\|\nabla f_{k}\right\|_{p_{k}} \leq\|\nabla p\|_{p} k^{-1+\frac{n}{p_{-}}} .
$$

Now, in order to prove $(2.17)$, observe that

$$
\int_{\mathbb{R}^{n}}\left|\nabla p_{k}\right|^{p_{k} r} d x=\int_{\mathbb{R}^{n}}|\nabla p|^{p r} k^{n-p r} d y
$$

As before,

$$
\int_{\mathbb{R}^{n}}|\nabla p|^{p r} k^{n-p_{+} r} d y \leq \int_{\mathbb{R}^{n}}\left|\nabla p_{k}\right|^{p_{k} r} d x \leq \int_{\mathbb{R}^{n}}|\nabla p|^{p r} k^{n-p_{-} r} d y
$$

Inequality (2.17) follows easily.

Remark 2.8. As observed before, the constant $\alpha(n, p)$ can be bounded below by a constant depending only on $n$ and $p_{+}$. Therefore, since $\left(p_{k}\right)_{+}=p_{+}$, it follows that $\alpha\left(n, p_{k}\right)$ is bounded below independently of $k$.

Remark 2.9. Take $\delta$ in $(2.14)$ so that the left hand-side is $\frac{\alpha(n, p)}{2}$. Since $\left(p_{k}\right)_{+}=p_{+}$, from $(2.14)$ we have

$$
\begin{aligned}
& \frac{\alpha\left(n, p_{k}\right)}{2} \leq C_{1, k}^{\frac{1}{p_{+}}} \max \left\{\left\|\mid \nabla p_{k}\right\|_{s}^{\frac{p_{-}}{p_{+}}},\left\|\nabla p_{k}\right\|_{s}\right\}+\frac{p_{+}}{n} \frac{\left\|\nabla f_{k}\right\|_{p_{k}}}{\left\|f_{k}\right\|_{p_{k}^{*}}}, \\
& C_{1, k}=2 \max \{\left(C(n, s) \operatorname{diam}\left(\operatorname{supp} f_{k}\right)\left\|1_{\text {supp } f_{k}}\right\|_{p^{\prime}} \frac{\left\|\nabla f_{k}\right\|_{p_{k}}}{\left\|f_{k}\right\|_{p_{k}^{*}}}\right)^{\frac{1}{\left(\frac{s}{s-p_{k}}\right)_{-}}} ; \\
&\left.\left(C(n, s) \operatorname{diam}\left(\operatorname{supp} f_{k}\right)\left\|1_{\text {supp } f_{k}}\right\|_{p^{\prime}} \frac{\left\|\nabla f_{k}\right\|_{p_{k}}}{\left\|f_{k}\right\|_{p_{k}^{*}}}\right)^{\frac{1}{\left(\frac{s}{s-p_{k}}\right)_{+}}}\right\}
\end{aligned}
$$

for all $f_{k} \in C_{c}^{\infty}\left(\mathbb{R}^{n}\right)$, and $k \in \mathbb{N}$ such that $p_{k}$ verifies condition (2.13). 
However, since $s>n$ it follows that $-p_{-}+\frac{n}{s}<0$ and so, by (2.17) there exists $k_{0}=$ $k_{0}\left(\delta, n, s,\left\||\nabla p|^{p}\right\|_{s}\right)$ such that $p_{k}$ verifies condition (2.13) for every $k \geq k_{0}$.

With all this preliminaries we are now in position to prove the main theorem of the section.

Proof of Theorem 1.1. We have already proved that the desired inequality holds if $\max \left\{\|\nabla p\|_{s}^{p_{-}},\|\nabla p\|_{s}^{p_{+}}\right\}$ is smaller than a fixed constant.

Now, by (2.17) and the fact that $s>\frac{n}{p_{-}}$, for any $p$ under our hypothesis we can fix $k>1$ large enough so that the Sobolev inequality holds for the exponent $p_{k}$. More precisely, it is enough to consider

$$
k=\max \left\{1, \frac{\max \left\{\|\nabla p\|_{s}^{p_{-}},\|\nabla p\|_{s}^{p_{+}}\right\}}{\delta}\right\},
$$

where $\delta$ is as in Remark 2.9, so that if $\max \left\{\|\nabla p\|_{s}^{p_{-}},\|\nabla p\|_{s}^{p_{+}}\right\} \leq \delta$, no scaling is needed. In this case, recall that the Sobolev inequality is just (2.14).

Let us now suppose that $\max \left\{\|\nabla p\|_{s}^{p_{-}},\|\nabla p\|_{s}^{p_{+}}\right\}>\delta$. Given $f \in C_{c}^{\infty}\left(\mathbb{R}^{n}\right)$ nonnegative such that $\|f\|_{p^{*}}=1$, the application of (2.15), (2.16) and the Sobolev inequality (2.21) for $p_{k}$ yield

$$
\begin{aligned}
& \frac{\alpha(n, p)}{2} \leq C\left(n, p_{+}, s\right) k^{\alpha\left(n, p_{+}, s\right)} \\
& \max \left\{\left(\operatorname{diam}(\operatorname{supp} f)\left\|1_{\text {supp } f}\right\|_{p^{\prime}}\|\nabla f\|_{p}\right)^{\frac{1}{\left(\frac{s}{s-p}\right)_{+}}},\right. \\
&\left.\left(\operatorname{diam}(\operatorname{supp} f)\left\|1_{\text {supp } f}\right\|_{p^{\prime}}\|\nabla f\|_{p}\right)^{\frac{1}{\left(\frac{s}{s-p}\right)_{+}}}\right\}^{\frac{1}{p_{+}}} \max \left\{\|\nabla p\|_{s}^{p_{-}},\|\nabla p\|_{s}^{p_{+}}\right\} \\
&+k^{\beta\left(n, p_{+}\right)} \frac{p_{+}}{n}\|\nabla f\|_{p} .
\end{aligned}
$$

Combining the previous inequality with the selection of $k$ we made, we can easily conclude the theorem.

\section{Trace inequality}

In this section we show the flexibility of mass transportation methods in dealing with Sobolevtype inequalities for variable exponents, applying the same type of arguments to the Sobolev trace inequality (1.4).

This method in the constant exponent case was first employed by Nazaret in [16] where, in addition, the author was able to compute the exact value of the optimal constant along with the extremals answering positively to a question raised by Escobar in [9].

Our arguments in this section follow closely the ones in [16] until some point where some new terms appear, due to the non constant nature of the exponent.

So, consider $f, g \in C_{c}^{\infty}\left(\mathbb{R}^{n}\right)$ nonnegative be such that such that $F:=f^{p^{*}}$ and $G:=g^{p^{*}}$ are probability densities in $\mathbb{R}_{+}^{n}$ and proceeding in the same way as in the previous section we obtain

$$
\int_{\mathbb{R}_{+}^{n}} g^{p_{*}} d x \leq \frac{1}{n} \int_{\mathbb{R}_{+}^{n}} F^{1-\frac{1}{n}} \Delta \varphi d x
$$


where $\Delta \varphi$ stands for the distributional laplacian of the convex function $\varphi$ such that $\nabla \varphi$ transport

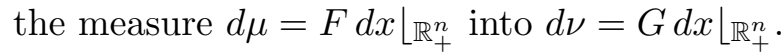

For technical reasons, it is convenient to subsitute $\varphi$ by a $\psi=\varphi-\mathbf{e} \cdot x$, where $\mathbf{e}=(-1,0, \ldots, 0)$ and since both functions $\varphi$ and $\psi$ have the same laplacian and we have that $\nabla \varphi \in B V_{\text {loc }}\left(\mathbb{R}_{+}^{n}\right)$, so we can apply the integration by parts formula for $B V$ functions and get

$$
\int_{\mathbb{R}_{+}^{n}} g^{p_{*}} d x \leq-\frac{1}{n} \int_{\mathbb{R}_{+}^{n}} \nabla\left(F^{1-\frac{1}{n}}\right) \cdot \nabla \psi d x-\frac{1}{n} \int_{\mathbb{R}^{n-1}} F^{1-\frac{1}{n}} d x^{\prime}+\frac{1}{n} \int_{\mathbb{R}^{n-1}} F^{1-\frac{1}{n}} \nabla \varphi \cdot \mathbf{e} d x^{\prime} .
$$

Since $\nabla \varphi \in \mathbb{R}_{+}^{n}$, it follows that $\nabla \varphi \cdot \mathbf{e} \leq 0$ on $\mathbb{R}^{n-1}$, so

$$
\int_{\mathbb{R}_{+}^{n}} g^{p_{*}} d x \leq-\frac{1}{n} \int_{\mathbb{R}_{+}^{n}} \nabla\left(F^{1-\frac{1}{n}}\right) \cdot \nabla \psi d x-\frac{1}{n} \int_{\mathbb{R}^{n-1}} F^{1-\frac{1}{n}} d x^{\prime}
$$

Up to know it is exactly the same as in Nazaret's paper [16]. See that paper for all the missing details.

Now is where the differences arise.

Proceeding as in the previous section we can estimate the first integral in the right-hand side,

$$
\begin{aligned}
-\frac{1}{n} \int_{\mathbb{R}_{+}^{n}} \nabla\left(F^{1-\frac{1}{n}}\right) \cdot \nabla \psi d x & \leq\left(\left(C\left(n, p_{+}, s, R\right)\|\nabla f\|_{p, \mathbb{R}_{+}^{n}}^{\alpha\left(n, p_{+}, s\right)}+C\left(n, p_{+}, s\right)\right) \max \left\{\|\nabla p\|_{s}^{p_{-}},\|\nabla p\|_{s}^{p_{+}}\right\}\right. \\
& \left.+C\left(n, p_{+}\right)\|\nabla f\|_{p, \mathbb{R}_{+}^{n}}\right) \max \left\{\left(\int_{\mathbb{R}^{n}} \tilde{\eta} G d y\right)^{\frac{1}{\left(p^{\prime}\right)_{-}}},\left(\int_{\mathbb{R}^{n}} \tilde{\eta} G d y\right)^{\frac{1}{\left(p^{\prime}\right)_{+}}}\right\},
\end{aligned}
$$

where $\tilde{\eta}(y)=\eta(y-\mathbf{e})=\max \left\{|y-\mathbf{e}|^{\left(p^{\prime}\right)_{+}},|y-\mathbf{e}|^{\left(p^{\prime}\right)_{-}}\right\}$.

Then, we reach the key estimate

$$
\begin{aligned}
\int_{\mathbb{R}^{n-1}} f^{p_{*}} d x^{\prime} & \leq\left(\left(C\left(n, p_{+}, s, R\right)\|\nabla f\|_{p, \mathbb{R}_{+}^{n}}^{\alpha\left(n, p_{+}, s\right)}+C\left(n, p_{+}, s\right)\right) \max \left\{\|\nabla p\|_{s}^{p_{-}},\|\nabla p\|_{s}^{p_{+}}\right\}\right. \\
& \left.+C\left(n, p_{+}\right)\|\nabla f\|_{p, \mathbb{R}_{+}^{n}}\right) \max \left\{\left(\int_{\mathbb{R}^{n}} \tilde{\eta} G d y\right)^{\frac{1}{\left(p^{\prime}\right)_{-}}},\left(\int_{\mathbb{R}^{n}} \tilde{\eta} G d y\right)^{\frac{1}{\left(p^{\prime}\right)_{+}}}\right\}-n \int_{\mathbb{R}_{+}^{n}} g^{p_{*}} d x,
\end{aligned}
$$

which is valid for all $f, g \in C_{c}^{\infty}\left(\mathbb{R}^{n}\right)$ nonnegative such that $\|f\|_{p^{*}, \mathbb{R}_{+}^{n}}=\|g\|_{p^{*}, \mathbb{R}_{+}^{n}}=1$.

Now, we denote by

$$
\beta(n, p)=\sup \frac{n \int_{\mathbb{R}_{+}^{n}} g^{p_{*}} d x}{\max \left\{\left(\int_{\mathbb{R}^{n}} \tilde{\eta} G d y\right)^{\frac{1}{\left(p^{\prime}\right)}-},\left(\int_{\mathbb{R}^{n}} \tilde{\eta} G d y\right)^{\frac{1}{\left(p^{\prime}\right)}+}\right\}}
$$

where the supremum is taken over all nonnegative $g \in C_{c}^{\infty}\left(\mathbb{R}^{n}\right)$ such that $\|g\|_{p^{*}, \mathbb{R}_{+}^{n}}=1$.

So, if we assume that $\max \left\{\|\nabla p\|_{s}^{p_{-}},\|\nabla p\|_{s}^{p_{+}}\right\}<\delta$ for some $\delta<\delta\left(n, p_{+}, s\right)$, namely $C\left(n, p_{+}, s\right) \delta<$ $\frac{1}{2} \beta(n, p)$, then

$C\left(n, p_{+}, s\right) \max \left\{\|\nabla p\|_{s}^{p_{-}},\|\nabla p\|_{s}^{p_{+}}\right\} \max \left\{\left(\int_{\mathbb{R}^{n}} \tilde{\eta} G d y\right)^{\frac{1}{\left(p^{\prime}\right)_{-}}},\left(\int_{\mathbb{R}^{n}} \tilde{\eta} G d y\right)^{\frac{1}{\left(p^{\prime}\right)_{+}}}\right\}-\frac{n}{2} \int_{\mathbb{R}_{+}^{n}} g^{p_{*}} d x<0$, 
for some $g \in C_{c}^{\infty}\left(\mathbb{R}^{n}\right)$ nonnegative with $\|g\|_{p^{*}, \mathbb{R}_{+}^{n}}=1$. Observe that at this point we require $p_{-}>1$. Therefore, for such $g$ we have

$\int_{\mathbb{R}^{n-1}} f^{p_{*}} d x^{\prime} \leq C\left(n, p_{+}, s, R\right) \max \left\{\|\nabla p\|_{s}^{p_{-}},\|\nabla p\|_{s}^{p_{+}}\right\}\|\nabla f\|_{p, \mathbb{R}_{+}^{n}}^{\alpha\left(n, p_{+}, s\right)}+C\left(n, p_{+}, s\right)\|\nabla f\|_{p, \mathbb{R}_{+}^{n}-B(n, p),}$

where $B(n, p)>0$.

The argument for a general exponent will be just as in Theorem 1.1, so without loss of generality we can assume that (3.2) holds.

Now, the Sobolev trace inequality (1.4) follows from (3.2) using the same scaling arguments as in [16]. We sketch these arguments for the reader's convenience.

Assume first that $\|f\|_{p_{*}, \mathbb{R}^{n-1}} \geq 1$, the other case is completely analogous. Then, from equation (3.2), it easily follows that

$$
Q(f)\left(\frac{\|\nabla f\|_{p}}{\|f\|_{p^{*}}}\right)^{\left(p_{*}\right)_{+}} \leq C_{1}\left(\frac{\|\nabla f\|_{p}}{\|f\|_{p^{*}}}\right)^{\alpha}+C_{2} \frac{\|\nabla f\|_{p}}{\|f\|_{p^{*}}}-B,
$$

where supp $f \subset B_{R}, C_{1}=C_{1}\left(n, p_{+}, s, R\right), C_{2}=C_{2}\left(n, p_{+}, s\right), \alpha=\alpha\left(n, p_{+}, s\right), B=B\left(n, p_{+}, p_{-}\right)$ and

$$
Q(f)=\frac{\int_{\mathbb{R}^{n-1}} f^{p_{*}} d x^{\prime}}{\|\nabla f\|_{p}^{\left(p_{*}\right)_{+}}} .
$$

Equivalently, considering $t=\frac{\|\nabla f\|_{p}}{\|f\|_{p^{*}}}$,

$$
Q(f) \leq\left(C_{1} t^{\alpha}+C_{2} t-B\right) t^{-\left(p_{*}\right)_{+}}=: h(t) .
$$

Now, it is easy to see that $h(t)$ is bounded above for $t>0$ for some constant depending on $C_{1}, C_{2}, B, \alpha$ and $p_{+}$which in turn depends on $n, p_{+}, s,\|\nabla p\|_{s, \mathbb{R}_{+}^{n}}$ and $R$.

We conclude that there exists some $C\left(n, p_{+}, s,\|\nabla p\|_{s, \mathbb{R}_{+}^{n}}, R\right)>0$ such that

$$
\int_{\mathbb{R}^{n-1}} f^{p_{*}} d x^{\prime} \leq C\|\nabla f\|_{p, \mathbb{R}_{+}^{n}}^{\left(p_{*}\right)_{+}}
$$

for all $f \in C_{c}^{\infty}\left(\mathbb{R}^{n}\right)$ satisfying $\|f\|_{p^{*}, \mathbb{R}_{+}^{n}} \geq 1$.

Analogously, if $f \in C_{c}^{\infty}\left(\mathbb{R}^{n}\right)$ is such that $\|f\|_{p^{*}, \mathbb{R}_{+}^{n}} \leq 1$, it follows that

$$
\int_{\mathbb{R}^{n-1}} f^{p_{*}} d x^{\prime} \leq C\|\nabla f\|_{p, \mathbb{R}_{+}^{n}}^{\left(p_{*}\right)_{-}} .
$$

Summing up, we have proved Theorem 1.2.

\section{ACKNOWLEDGMENTS}

This paper was partially supported by Universidad de Buenos Aires under grant UBACyT 20020130100283BA, by CONICET under grant PIP 2009 845/10 and by ANPCyT under grant PICT 2012-0153. J. Fernández Bonder and A. Silva are members of CONICET and J.P. Borthagaray is a doctoral fellow of CONICET. 


\section{REFERENCES}

[1] Robert A. Adams. Sobolev spaces. Academic Press [A subsidiary of Harcourt Brace Jovanovich, Publishers], New York-London, 1975. Pure and Applied Mathematics, Vol. 65.

[2] Yann Brenier. Décomposition polaire et réarrangement monotone des champs de vecteurs. C. R. Acad. Sci. Paris Sér. I Math., 305(19):805-808, 1987.

[3] Yann Brenier. Polar factorization and monotone rearrangement of vector-valued functions. Comm. Pure Appl. Math., 44(4):375-417, 1991.

[4] D. Cordero-Erausquin, B. Nazaret, and C. Villani. A mass-transportation approach to sharp Sobolev and Gagliardo-Nirenberg inequalities. Adv. Math., 182(2):307-332, 2004.

[5] Lars Diening. Riesz potential and Sobolev embeddings on generalized Lebesgue and Sobolev spaces $L^{p(\cdot)}$ and $W^{k, p(\cdot)}$. Math. Nachr., 268:31-43, 2004.

[6] Lars Diening, Petteri Harjulehto, Peter Hästö, and Michael Růžička. Lebesgue and Sobolev spaces with variable exponents, volume 2017 of Lecture Notes in Mathematics. Springer, Heidelberg, 2011.

[7] David E. Edmunds and Jiří Rákosník. Sobolev embeddings with variable exponent. Studia Math., 143(3):267293, 2000.

[8] David E. Edmunds and Jiří Rákosník. Sobolev embeddings with variable exponent. II. Math. Nachr., 246/247:53-67, 2002.

[9] José F. Escobar. Conformal deformation of a Riemannian metric to a scalar flat metric with constant mean curvature on the boundary. Ann. of Math. (2), 136(1):1-50, 1992.

[10] Lawrence C. Evans and Ronald F. Gariepy. Measure theory and fine properties of functions. Studies in Advanced Mathematics. CRC Press, Boca Raton, FL, 1992.

[11] Xianling Fan. Boundary trace embedding theorems for variable exponent Sobolev spaces. J. Math. Anal. Appl., 339(2):1395-1412, 2008.

[12] Petteri Harjulehto and Peter Hästö. Sobolev inequalities with variable exponent attaining the values 1 and n. Publ. Mat., 52(2):347-363, 2008.

[13] L. V. Kantorovich. On a problem of monge (in russian). Uspekhi Mat. Nauk., 3:225-226, 1948.

[14] Robert J. McCann. Existence and uniqueness of monotone measure-preserving maps. Duke Math. J., 80(2):309-323, 1995.

[15] Gaspard Monge. Mémoire sur la théorie des déblais et des remblais. Histoire de l'Académie Royale des Sciences de Paris, pages 666-704, 1781.

[16] Bruno Nazaret. Best constant in Sobolev trace inequalities on the half-space. Nonlinear Anal., 65(10):19771985, 2006.

[17] Cédric Villani. Topics in optimal transportation, volume 58 of Graduate Studies in Mathematics. American Mathematical Society, Providence, RI, 2003.

[18] Cédric Villani. Optimal transport, volume 338 of Grundlehren der Mathematischen Wissenschaften [Fundamental Principles of Mathematical Sciences]. Springer-Verlag, Berlin, 2009. Old and new.

(J.P. Borthagaray and J. Fernández Bonder) IMAS - CONICET and Departamento de MatemáticA, FCEyn - Universidad de Buenos Aires, Ciudad Universitaria, Pabellón I (1428) Buenos Aires, ArGENTINA.

(A. Silva) IMASL - CONiCET and Departamento de Matemática, Universidad Nacional de San Luis (5700) SAN Luis, Argentina

E-mail address, J.P. Borthagaray: jpbortha@dm.uba.ar

E-mail address, A. Silva: acsilva@unsl.edu.ar

E-mail address, J. Fernández Bonder: jfbonder@dm.uba.ar

URL, J. Fernández Bonder: http://mate.dm.uba.ar/ j jfbonder 